\title{
XVIII. Electromagnetic and calometric absolute measurements: the absolute value of Siemens's unit of resistance in electromagnetic measure; the relation between the current-work and the heat-evolution in stationary galvanic currents; and the absolute values of some constant hydroelectromotive forces in electromagnetic measure. (Condensed comparison of the results of a series of investigations.)
}

\section{H. F. Weber}

To cite this article: H. F. Weber (1878) XVIII. Electromagnetic and calometric absolute measurements: the absolute value of Siemens's unit of resistance in electromagnetic measure; the relation between the current-work and the heat-evolution in stationary galvanic currents; and the absolute values of some constant hydroelectromotive forces in electromagnetic measure. (Condensed comparison of the results of a series of investigations.) , Philosophical Magazine Series 5, 5:29, 127-139, DOI: 10.1080/14786447808639398

To link to this article: http://dx.doi.org/10.1080/14786447808639398

曲 Published online: 13 May 2009.

Џ Article views: 1
Submit your article to this journal 주

View related articles 
of a gas regulate their motions so as to move in a particular nanner, though we doubt whether, if we had not arrived at this conclusion independently for ourselves, we should have been able to make a practical application of it. The point it has been our object to call attention to (and which apparently has not been noticed by others) is, that the motion of the particles of a gas willin the range of free path precisely satisfies all the conditions Le Sage arbitrarily assuned in order to produce gravity-or that the special character of the motion Le Sage arbitrarily assumed his streams of particles to have, actually exists within the range of free path of the particles of a gas -in other words, that all the effects of gravity can be produced by the mere existence of a gas in space, and indeed mast be produced if such a gas exists.

XVIII. Electromagnetic and Calometric Absolute Measurements: the Absolute Value of Siemens's Unit of Resistance in Electromagnetic Measure; the Relation between the Current-worle and the Heat-evolution in stationary Galvanic Currents; and the Absolute Values of some constant Hydroelectromotive Forces in Electromagnetic Measure. (Condensed Comparison of the Results of a Series of Investigations.) By H. F. Weber, Professor of Mathematical and Technical Physics at the Federal Polytechnic Academy of Zurich.

[Continued from p. 43.]

\section{The Heat produced by Stationary Galvanic Currents.}

MR. JOULE, thirty-seven years since, showed by experiI ment that the quantity of heat which a stationary galvanic current of intensity $i$ generates in a conductor whose resistance is $w$, during the time $z$, is proportional to $i^{2} w z$. Sir W. Thomson then, in 1851 (and Prof. Clausius and others later), proved in the theoretical way that the value of the mechanical work which is expended in the stationary galvanie current of the intensity $i$, in a conductor with the resistance $w$, along which the electromotive force $\mathrm{E}$ is in action, in the time $z$ is equal to the product $i \mathrm{E} z$, or, pursuant to Ohm's law, equal to the expression $i^{2} w z$, where the quantities $\mathrm{E}, i, w$ are to be taken as measured according to absolute measure. If we make the assumption that, in a stationary galvanic current in which the evolution of heat is the only action of the currentflow, the amount of heat developed in the unit of time, $Q$, is the full equivalent of the work expended in the same time, then we have

$$
\mathrm{JG}=i^{2} w=i \mathrm{E},
$$

where $J$ denotes the mechanical equivalent of the unit of heat. 
On this hypothesis, consequently, the proportionality-factor of Joule's law of heat-evolution is equal to the reciprocal value of J. Supposing that this assumption, the whole of the mechanical work consumed by the stationary galvanic current appears in the form of heat, is correct, we have a new definition for the absolute resistance of a conductor :-The absolute resistance (measured according to any system) of a conductor is equal to the mechanical value of the amount of heat which is generated in the conductor in the unit of time by the constant galvanic current 1 (measured according to the same system of measurement). And a new method for the experimental determination of the absolute resistance of a conductor:Measure the amount of heat, $\mathrm{Q}$, which in the time $z$ is generated by the constant current $i$ measured in absolute measure ; then the absolute value of the resistance (measured in the same system of measurement in which $i$ is measured) is

$$
w=\frac{\mathrm{JQ}}{i^{2} z} \text {. }
$$

It cannot be maintained that the correctness of the hypothesis, "in the stationary galvanic current the entire work of the current is converted into heat," is so far above all doubt that one can without hesitation make use of the heat developed in a conductor by the stationary galvanic current for the absolute measurement of the resistance of the conductor. The results of the most exact investigations which have yet been instituted in this direction for testing the fundamental hypothesis in question contradict one another. Von Quintus Icilius (Pogg. Ann. vol. ci. 1856), in a carefully executed very extended series of operations, obtained the final result that the stationary galvanic current develops about 7 per cent. more heat than it should according to Thomson's equation ; on the other hand, Joule *, in a comprehensive and very accurately executed investigation, with which he was charged by the British-Association Committee for the production of standards of resistance, found by experiment that in fact almost as much heat is produced in a conductor by the stationary galvanic current as is specified by the above-mentioned law. Von Quintus Icilius calculates from his experiments the mechanical equivalent of the unit of heat to be 399.7 metrekilograms ; Joule infers from his the value 429.3 metre-kilograms for $J$ (expressed in the usual measure of mechanical work). While the discrepancy between the results of these two series of observations is not cleared up the galvanic heat-

* Reports of Electrical Standards, edited by Jenkin, p. 165. 
development cannot be unhesitatingly made use of for the absolute determination of resistance.

In order to procure the means to enable me to carry out an entirely unexceptionable determination of the absolute value of the S. M. U. by the heat-evolution of stationary galvanic currents, I undertook, in a very extensive and in many ways varied third experimental investigation, as rigorously exact a solution as possible of the question :-In a stationary galvanic current in which evolution of heat appears as the only action, is the heat generated in a certain time the exact equivalent of the mechanical work consumed by the current during that time?

In the path of a current maintained constant, of which the absolute intensity $i$ was carefully measured electromagnetically, was placed a thin platinum wire of about $15 \mathrm{~S}$. M. U. resistance, wound in zigzag upon a numerously perforated frame of hardgum. Thick copper wires conducted the current to and from the platinum wire. The frame carrying the wire was suspended in a water calorimeter of the thinnest sheet copper, which was in an environment of constant temperature. The water filling the calorimeter amounted to about 250 grams; the water-worth of the calorimeter-vessel, the frame, and the thermometer amounted to about 3 grams.

The constant current with the intensity $i$ was conducted, during the time $z$, through the resistance $w$ in the calorimeter. The mechanical work consumed by the current during this time, within the conductor with the resistance $w$, was then $i^{2} w z$. On the other hand, a certain amount of heat $Q$ was generated in the resistance $w$, was given up to the calorimeter, and was to be calculated from the rises of temperature in the calorimeter, the water-worths of the substances filling the calorimeter, and the losses of heat of the calorimeter by radiation outwards or the gain of heat by the calorimeter from without. The mechanical value of this amount of heat, JQ, would necessarily, if the total work of the current were converted into heat, be equal to $i^{2} w z$.

On the hypotheses that the entire work of the current is converted into heat, that the exchange of heat between the calorimeter and its surrounding is governed by Newton's law, that the specific heat of water increases linearly with the temperature, and that the resistance of the platinum wire used increases proportionally with the temperature, the following differential equation holds for the dependence of the variable temperature $t$ of the calorimeter on the time $z$ :-

$$
\mathrm{M} c_{a}\left[1+\gamma\left(t-t_{a}\right)\right] d t=\frac{i^{2} w_{a}}{\mathrm{~J}}\left[1+q\left(t-t_{n}\right)\right] d z-h\left(t-t_{a}\right) d z .
$$

Phil. Mag. S. 5. Vol. 5. No. 29. Feb. 1878. 
In this equation, $M$ denotes the sum of the water-worths of the substances filling the calorimeter, $t_{a}$ the constant temperature of the environment of the calorimeter ; $c_{a}$ the specific heat of water, and $w_{a}$ the absolute resistance of the platinum wire, at the temperature $t_{a} ; \gamma$ the coefficient of the increase of the specific heat of water, and $q$ the coefficient of the increase of the absolute resistance of the platinum wire, for $1^{\circ}$ rise of temperature; and $h$ the heat which the calorimeter would part with to the outside if its temperature were $1^{\circ}$ higher than that of its environment.

If we put $\mathrm{A}=\frac{i^{2} w_{a}}{\mathrm{JM} c_{a}}$ and $\mathrm{B}=\frac{\mathrm{J} h-(q-\gamma) i^{2} w_{a}}{\mathrm{JM} c_{a}}$ and assume that at the time $z=0$ the temperature of the calorimeter is equal to $t_{0}$, the integration of the above differential equation gives the following connexion between the variable temperature $t$ of the calorimeter and the time $z$ :-

$$
t-t_{0}=\left(\frac{\mathrm{A}}{\mathrm{B}}-t_{0}+t_{a}\right)\left(1-e^{-\mathrm{Bz}}\right) ; . . .
$$

or if the notion "mean temperature of the calorimeter during the time $z=0$ to $z=z$ " be introduced with the symbol $\bar{t}$,

$$
\mathrm{JM} c_{a}\left[t-t_{0}+\mathrm{B}\left(\bar{t}-t_{a}\right) z=i^{2} w_{a} z . . . .\right.
$$

The quantity $\mathrm{B}\left(\bar{t}-t_{a}\right) z$ represents the temperature-correction which must be applied to the direct reading of the rise of temperature of the calorimeter on account of the heat-exchange with the environment, and on account of the variability of the resistance as well as that of the specific heat of water with rising temperature. This correction can be made as small as we please, by a suitable selection of the quantity $\bar{t}-t_{x}$. In all the measurements executed, care was taken so that this difference only amounted to so small a fraction of a degree that the correction, $\mathrm{B}\left(t-\bar{t}_{a}\right) z$, to be added to $t-t_{0}$ amounted to only from $\frac{1}{300}$ to $\frac{\lambda}{200}$ of $t-t_{0}$. The period $z$ was chosen so great that the rise of temperature amounted to about $15^{\circ}$. For the determination of the mean temperature $\bar{t}$ of the calorimeter, and of the constant $B$, the temperature of the calorimeter was read off, from the commencement onwards, every five minutes; in this way a series of equations of the form (1) were obtained, from which $B$ could be ascertained. The thermometer of the calorimeter was most carefully compared, within its entire scale, with the air thermometer; all readings taken from it were always reduced to the indications of the latter instrument. 
The current-intensity $i$ was measured in absolute electromagnetic measure by means of the already mentioned simple tangent-compass ( $R=165.7$ millims.) according to the relation

$$
i=\frac{\mathrm{RH}}{2 \pi}\left(1-\frac{3}{4} \frac{l^{2}}{\mathrm{R}^{2}}\right)(1+\theta) \tan u ;
$$

for the measurement of $u$, mirror, telescope, and scale were made use of. To eliminate the daily variations of $\mathrm{H}$, which on some days may reach $\frac{1}{5}$ per cent. of the mean value, $H$ was determined, for the place of the tangent-compass, before and after each measurement. The variations of declination of the earth's magnetic force (which towards noon are very considerable for delicate measurements) were eliminated by regularly recurring, rapidly executed reversals of the current. A very powerful damper enveloped the magnetic needle of the compass, and permitted the readings of the deviations of the magnet to be taken again 20 seconds after the reversal of the current. The intensity of the current was maintained constant within $\frac{1}{500}$ or $\frac{1}{600}$ of its value by aid of a Dubois-Reymond's rheochord in the path of the current. The quantities $l$ and $\theta$ were so small that the sum of the two corrections, $-\frac{3}{4} \frac{l^{2}}{\mathrm{R}^{2}}+\theta$, amounted to only $+0 \cdot 0008$.

The absolute value of the resistance $w$ was determined by the method described above in section II. As the temperature $t_{a}$ of the calorimeter-environment varied somewhat from one day to another (up to $3^{\circ}$ ), the coefficient of the increase of the resistance for $1^{\circ}$ of rise of temperature had also to be known. To obtain the latter the absolute value of the resistance $w$ was determined for the two temperatures (maintained constant) $0^{\circ}$ and $23^{\circ}$. At the same time the value of $w$ for the same temperatures was measured in relative Siemens measure. The resistance of the platinum wire was found as follows:-

Tempera- In absolute measure. In relative measure. Date.
ture. $22.514 \cdot 498 \times 10^{10}\left(\frac{\text { millim. }}{\text { sec. }}\right) \quad 15 \cdot 141$ S. U. Oct. 14,1876 .

\begin{tabular}{|c|c|c|c|c|c|}
\hline $\begin{array}{l}22 \cdot 9 \\
23 \cdot 7\end{array}$ & $\begin{array}{l}14.419 \times 10^{10} \\
14^{\circ} 486 \times 10^{10}\end{array}$ & " & $\begin{array}{l}15 \cdot 142 \\
15 \cdot 154\end{array}$ & " & $\begin{array}{l}" 15, \\
" 16,\end{array}$ \\
\hline $\begin{array}{l}0 \\
0 \\
0\end{array}$ & $\begin{array}{l}14 \cdot 141 \times 10^{10} \\
14 \cdot 121 \times 10^{10} \\
14 \cdot 130 \times 10^{10}\end{array}$ & $"$ & $\begin{array}{l}14 \cdot 782 \\
14 \cdot 791 \\
14 \cdot 770\end{array}$ & " & $\begin{array}{l}" 17, \\
" 18, \\
" 19,\end{array}$ \\
\hline
\end{tabular}


For $23^{\circ}$ the platinum wire possesses the absolute resistance

and the relative resistance

$$
14 \cdot 468 \times 10^{10}\left(\frac{\text { millim. }}{\text { sec. }}\right)
$$

$$
15 \cdot 146 \text { S. M. U. ; }
$$

and for $0^{\circ}$ the platinum wire possesses the absolute resistance

and the relative resistance

$$
14 \cdot 131 \times 10^{10}\left(\frac{\text { millim. }}{\text { sec. }}\right) \text {, }
$$

\section{$14 \cdot 781$ S. M. U.}

From the first result it follows that

from the latter,

$$
1 \text { S. M. U. }=0.9552 \times 10^{10}\left(\frac{\text { millim. }}{\text { sec. }}\right) \text {; }
$$

$$
1 \text { S. M. U. }=0.9560 \times 10^{10}\left(\frac{\text { millim. }}{\text { sec. }}\right) \text {,- }
$$

which are in perfect harmony with the results previously obtained in sections I. and II. We obtain the coefficient of the increase of the resistance, referred to $1^{\circ}$ of temperatureincrease :-

$$
\left.\begin{array}{l}
\text { From the absolute measurements, } \quad q=0 \cdot 001035 . \\
\text { And from the relative measurements, } q=0 \cdot 001074 .
\end{array}\right\}
$$

For the temperature $t_{a}$, employed in the experiment in question, the absolute value $w_{a}$ was calculated according to the formula

$$
w=14 \cdot 131[1+0 \cdot 001054 t] \times 10^{10}\left(\frac{\text { millim. }}{\text { sec. }}\right) .
$$

From the results adduced it follows that absolute determinations of resistance can be accomplished with such precision that the variability of the resistance with varying temperature can be ascertained from them very nearly as accurately as from resistance-comparisons according to the bridge-method:

In the course of the investigation a gradual alteration of the resistance of the platinum by the continual passage of currents through it was sought after with peculiar care. On the 16 th October, 1876 , at the temperature of $23^{\circ} .7$ the resistance was found equal to $15 \cdot 154 \mathrm{~S} . \mathrm{M} . \mathrm{U}$., or, reduced to $16^{\circ}$, equal to $15^{\prime} 032$ S. M. U.

After the wire had served for twelve experiments, in which a current of absolute intensity 4 (a round number) passed through it:during about an hour, it showed, on the 19th De- 
cember, 1876, a resistance of $15.068 \mathrm{~S}$. M. U., or, reduced to the temperature $16^{\circ}$, of $15^{\circ} 035 \mathrm{~S} . \mathrm{M}$. U. At the end of twelve more experiments, in which a current of about 6 absolute units passed through the wire each time during about $45 \mathrm{mi}$ nutes, the latter showed, on the 28th March, at the temperature of $16^{\circ}$, the resistance $15^{\circ} 031 \mathrm{~S} . \mathrm{M}$. U.

Accordingly, under the influence of continual currents of absolute intensity from 4 to 6 the platinum wire underwent no demonstrable alterations. A special investigation showed that perceptible permanent alterations in the resistances of metallic conductors only make their appearance from a definite current-intensity onwards.

Without further remarks, the following are the results of the investigation.

Here also I varied the experiments in several ways. First, a series of twelve observations was instituted in which a proportionally feeble current passed through the wire in the calorimeter during a proportionally long period. From these twelve observations the following values were obtained for the mechanical equivalent of the unit of heat (the numbers are based on the ordinary measure of work; and with each is given the external temperature $t_{a} \cdot$ to which the heat-unit on which the result is based refers):-

\begin{tabular}{|c|c|c|c|c|c|}
\hline \multicolumn{3}{|c|}{ Date. } & \multicolumn{2}{|r|}{$t_{a}}$. & $\underset{\text { metre-kilograms }}{\mathrm{J} .}$ \\
\hline October & 20,1 & 1876 & & $16 \cdot 6$ & $428 \cdot 49$ \\
\hline " & 21 & " & .......... & 16.7 & $428 \cdot 12$ \\
\hline$"$ & 26, & $"$ & .......... & $16 \cdot 3$ & $425 \cdot 51$ \\
\hline$"$ & 28, & , & .......... & $18 \cdot 1$ & 426.93 \\
\hline$"$ & 30 & , & $\cdots .$. & $18 \cdot 5$ & $429 \cdot 93$ \\
\hline N" & 31 & " & .......... & $18 \cdot 0$ & $429 \cdot 56$ \\
\hline Novembe & 5 & " & .......... & $16 \cdot 2$ & $428 \cdot 18$ \\
\hline$"$ & 6 & $\eta$ & .......... & $16 \cdot 0$ & $427 \cdot 28$ \\
\hline$"$ & 9, & $"$ & .......... & $16 \cdot 4$ & $426 \cdot 95$ \\
\hline$"$ & 15, & " & ........... & $17 \cdot 1$ & 428.50 \\
\hline$"$ & 10 & & & 0 & 42640 \\
\hline & 20, & & & & \\
\hline
\end{tabular}

Hence the mean mechanical equivalent $J$ of the heat-unit is equal to 42776 metre-kilograms (with a probable error of $\pm 0.23)$, if the specific heat of water at the mean temperature employed, $\bar{t}_{a}=17^{\circ} \cdot 2$, be put $=1$.

A second series of twelve measurements was next instituted, in which a proportionally stronger current was employed during a shorter time. The results obtained in this series were:- 


\begin{tabular}{|c|c|c|c|c|c|}
\hline \multicolumn{3}{|c|}{ Date. } & \multicolumn{2}{|r|}{$t_{a}$} & J. \\
\hline Deceml & r 21, & 1876 & ..... & 198.8 & $\begin{array}{c}\text { metre-k1ograms. } \\
428 \cdot 36\end{array}$ \\
\hline 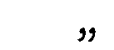 & 22 , & $"$ & .......... & $19 \cdot 7$ & $430 \cdot 31$ \\
\hline " & 23 , & ", & .......... & $18 \cdot 7$ & $426 \cdot 37$ \\
\hline " & 24 , & $"$ & ......... & $18 \cdot 8$ & $427 \cdot 50$ \\
\hline$"$ & 25 , & g & .......... & $18 \cdot 8$ & $427 \cdot 45$ \\
\hline , & 26 , & " & $\ldots \ldots \ldots$ & $20 \cdot 0$ & $429 \cdot 18$ \\
\hline " & 27, & " & .......... & $20 \cdot 1$ & $428 \cdot 02$ \\
\hline , & 28, & $"$ & •........ & $19 \cdot 9$ & $429 \cdot 87$ \\
\hline$"$ & 29 & " & •......... & $19 \cdot 4$ & $430 \cdot 15$ \\
\hline$"$ & 30 , & , & ......... & $19 \cdot 7$ & $426 \cdot 93$ \\
\hline Janı & 31, & $18 \ddot{87}$ & 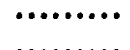 & $\begin{array}{l}19 \cdot 5 \\
19 \cdot 6\end{array}$ & $\begin{array}{l}427 \cdot 90 \\
498 \cdot 96\end{array}$ \\
\hline
\end{tabular}

According to this the mean mechanical equivalent of the heatunit is equal to $428^{\prime} 42$ metre-kilograms (with the probable error $\pm 0^{\circ} 25$ ), the specific heat of water at the mean temperature $\bar{t}_{a}=19^{\circ} .5$ employed being put $=1$.

In a third series of experiments the period and the currentintensity were chosen such that the rise of temperature in the calorimeter amounted, as in the previous experiments, to aboul $15^{\circ}$. The proportions, however, were not so closely limited as to make the difference $\vec{t}-t_{n}$ as small as possible; rather a play of a few degrees was given to it. The results of this series, in which the exchange of heat between the calorimeter and its environment possessed a value four or five times as great as in the previous series, were :-

\begin{tabular}{|c|c|c|c|c|}
\hline \multicolumn{3}{|c|}{ Date. } & $t_{\alpha}$ & J.. \\
\hline March & 28 , & 1877 & ........ $16^{\circ} \cdot 1$ & $427 \cdot 15$ \\
\hline " & 29 , & , & ......... $16 \cdot 6$ & $429 \cdot 30$ \\
\hline " & 30 & 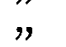 & ........ $16 \cdot 8$ & $429 \cdot 61$ \\
\hline & 31 , & , & ......... $17 \cdot 3$ & $428 \cdot 03$ \\
\hline pril & 1 & $\eta$ & $\ldots 17 \cdot 0$ & 426.92 \\
\hline$"$ & 2, & $\eta$ & .......... $17 \cdot 7$ & $428 \cdot 56$ \\
\hline " & 3 , & $"$ & ......... $18 \cdot 3$ & $427 \cdot 91$ \\
\hline " & 4 & $"$ & …...... $18 \cdot 0$ & $429 \cdot 10$ \\
\hline$"$ & 5 , & $"$ & ........ $17 \cdot 7$ & $427 \cdot 85$ \\
\hline " & 6 , & $"$ & ......... 18.9 & $427 \cdot 52$ \\
\hline " & 7 & $"$ & ......... 18.5 & $428 \cdot 43$ \\
\hline " & 8, & " & ......... $17 \cdot 9$ & $428 \cdot 93$ \\
\hline
\end{tabular}

According to this series the mean value of the mechanical equivalent of the unit of heat is $4^{2} 8^{\circ} 28$ metre-kilograms (with a probable error of $\pm 0^{\prime} 18$ ), the specific heat of water, at the mean temperature $\bar{t}_{n}=17^{\circ} .6$ employed, being put $=1$. 
As the general result of these 36 tolerably accordant experiments (the extremes differing at the most only $\frac{1}{2}$ per cent. from the mean) we get:- The mechanical equivalent of the heat-unit, derived from the heat-evolution of the stationary galvanic current, has the value $428^{\prime} 15$ metre-kilograms (with the probable error $\pm 0^{*} 22$ ), understanding by unit of heat that amount which must be supplied to the anit of mass (1 kilogram) of water in order to raise its temperature $1^{\circ} \mathrm{C}$. as measured by the air thernometer.

The surest means for deriving, in a purely thermic way, the quantitative value of the mechanical equivalent of the heatunit is unquestionably furnished by the relation between the two specific heats of an ideal permanent gas--

or

$$
\mathrm{J}\left(c_{p}-c_{v}\right)=p_{0} v_{0} \alpha,
$$

$$
\mathrm{J}_{c_{p}} \frac{k-1}{k}=p_{0} v_{0}{ }^{\alpha} \text {. }
$$

For atmospheric air the three quantities $p_{0} v_{0}, \alpha$, and $c_{p}$ are very accurately known from Regnault's measurements : $p_{0} v_{0}=7991$; $\alpha=0.00367$; and $c_{p}=0.23754$. The quantity $k$ has been more recently determined for the same gas very carefully by $M$. Röntgen: $k=1 \cdot 4053$. Inserting these numerical values in the last equation, and also taking into account that, according to the experiments of Joule and Thomson, atmospheric air accomplishes in alterations of volume, besides the external work performed, an internal work equal to about $\frac{1}{300}$ of the external, we obtain from the thermal behaviour of air 428.95 metrekilograms as the mechanical equivalent of the unit of heat. The unit on which this number is based is that quantity of heat which must be supplied to the mass-unit (1 kilogram) of water at $14^{\circ}$ or $15^{\circ}$ in order to bring about a rise of temperature of $1^{\circ}$ (measured by the air thermometer).

Dr. Joule, in 1849, noted as the most trustworthy result of his numerous experiments on friction for the determination of the mechanical equivalent of the unit of heat the value $J=423.79$ metre-kilograms. In the calculation of this number the specific heat of water was put $=1$ for the temperature $14^{\circ} .4$; moreover the specific heat of the calorimeter-vessel was assumed too high. If the necessary correction on account of the latter circumstance be added, the result just mentioned becomes $\mathrm{J}=424 \cdot 39$ metre-kilograms. The sixty frictionexperiments made quite recently by Joule have given almost exactly the same result, $424 \cdot 67$ metre-kilograms.

Unfortunately the total result of Joule's friction-experiments, 
$\mathrm{J}=424.50 \mathrm{~m} .-\mathrm{k}$, cannot be compared at once with that obtained from the behaviour of gases, $\mathrm{J}=428.95 \mathrm{~m} .-\mathrm{k}$. The two values are referred to quite different units: the basis of the former is $1^{\circ}$ of Joule's mercury thermometer; that of the latter is $1^{\circ}$ of the air thermometer. These two units may possibly differ 1 per cent. Perhaps, on reducing his previous and his recent friction-experiments to the indications of the air thermometer, Dr. Joule obtains a final result as good as identical with the value of $J$ that follows from the behaviour of gases.

On account of this disturbing circumstance, I hold the value $\mathrm{J}=428 \cdot 95$, drawn from the behaviour of gases, and immediately comparable with my above result, to be the most certain of those given by purely thermal determinations. Consequently, from the heat-evolution of stationary galvanic currents there comes as good as the same mechanical equivalent of the heat-unit as from purely thermal processes*. The hypothesis that the entire work consumed in the stationary current-flow appears in the form of heat has verified itself.

There still remains to say a few words on the already mentioned determinations, carried out by Joule and Von Quintus Icilius, of the mechanical equivalent. of the heat-unit by galvanic heat-evolution.

Dr. Joule carried out 45 experiments, in three series $\dagger$. He regards as the most trustworthy result that of the last series, comprising 30 experiments- $\mathrm{J}=429 \cdot 3 \mathrm{~m} .-\mathrm{k}$. In the calculation of this number the specific heat of water at $18^{\circ} \cdot 4$ was put $=1$, and it was further assumed that the British unit of resistance in fact possesses the asserted value $10^{10}$ ( $\left.\frac{\text { millim. }}{\text { sec. }}\right)$. According to our results this is not quite exactly the case : if the ratio of the British unit to Siemens's is as $1: 0.9536$, then the absolute value of the former is $=1.0014 \times 10^{10}$ ( $\left.\frac{\text { millim. }}{\text { sec. }}\right)$,

- The two results, $J=428 \cdot 15$ (derived from the galvanic evolution of heat) and $J=428.95$ (determined from the thermal behaviour of the permanent gases), refer, as was expressly remarked, to two different units of heat: in the former the unit is that quantity which can heat the unit of mass of water from $17^{\circ} .5$ to $18^{\circ} .5$; in the latter it is that which can heat the mass-unit of water from $14^{\circ}$ to $15^{\circ}$. Therefore the two results will only then be strictly comparable, when the variation of the specific heat of water at variable temperature is certainly known. The experiments which I have, up to the present, instituted for fixing this hitherto totally uncertain quantity are not yet brought to a perfectly satisfactory conclusion. Yet so much can be positively known, that the reduction of the two values of $J$ to the same temperature will bring about only a very slight alteration.

† Reports of Electrical Standards, edited by Jenkin, p. 175. 
and Joule's result becomes $429.9 \mathrm{~m} .-\mathrm{k}$. Unfortunately, in this measurement also, Joule took for the basis the degree of the mercury thermometer, and not that of the air thermometer, and thereby made a precise comparison of his final result with ours impossible. Thus much may, however, be regarded as established, that so soon as Joule's mercury thermometer does not differ very considerably from the air thermometer, a tolerably good accordance exists between the results of the measurements made by Dr. Joule and by myself.

Von Quintus Icilius did not gauge the resistances made use of in his numerous measurements* according to absolute measure. The absolute resistance-values which formed the basis of his calculations he ascertained by a comparison of his resistances with the second copy of Jacobi's resistance-unit, produced by Wilhelm Weber for himself, and gauged by him according to its absolute value. This copy of Jacobi's standard was $=0.9839$ of Jacobi's unit; and since, according to W. Weber's absolute-resistance measurements, the absolute value of Jacobi's resistance-unit is $=0.598 \times 10^{10}\left(\frac{\text { millim. }}{\text { sec. }}\right)$, the copy had the absolute value $0.5884 \times 10^{10}\left(\frac{\text { millim. }}{\text { sec. }}\right)$. Von Quintus Icilius regards as the most trustworthy of his experiments the 34 in which water was employed as the calometric liquid. From these 34 experiments he calculates, as the final result, $\mathrm{J}=399^{\circ} 7$ m.-k. Singularly, this result has not in the least aroused the attention of physicists; and yet it was to be inferred therefrom, either that the measurements which conducted to it were very faulty, or that the theoretical views which formed its basis needed correction. The essentially different result obtained by me, in which by repeated trials I could detect no error, and the good accordance of which with Joule's results I could not but consider a further sign of its approximate correctness, caused me to reflect long upon the cause of the discrepancy; at last $I$ succeeded in attaining a complete explanation:$W$.Weber, in his first absolute-resistance determination, found for the absolute value of Jacobi's resistance-unit about $8 \mathrm{per}$ cent. too small a number, in consequence of which Quintus Icilius's final result could not but come out just as much too little. If this error be corrected, the latter value (399.7 m.-k.) becomes $431^{\circ} 6 \mathrm{~m} .-\mathrm{k}$., a value which, certainly, is somewhat greater than that which results from Joule's measurements and my own experiments; but taking into consideration that Quintus Icilius has quite neglected the variation of the hori-

* Pogg. Ann. vol. ci. p. 65. 


\section{Electromagnetic and Calometric Absolute Measurements.}

zontal component of the earth's magnetic force (which alone might make a difference of 2 units in the value of $J$ ), that he has not reduced the indications of the thermometer he employed to the air thermometer (a reduction which might make a difference of 4 units), and that he used in his experiments very powerful currents and very feeble resistances (a procedure which must necessarily have been attended with some slight errors), no great weight will be laid upon this small difference; the previously startling discrepancy is removed.

It can in two ways be shown that W. Weber, as we have maintained, found the absolute value of Jacobi's unit of resistance about 8 per cent. too small.

Bosscha, in 1856*, determined according to Ohm's method the electromotive force of a Daniell's element in absolute electromagnetic measure. His measurements were based on a standard of resistance the absolute value of which, $0.607 \times 10^{10}\left(\frac{\text { millim. }}{\text { sec. }}\right)$, was obtained by comparison with the above-mentioned copy by W. Weber of Jacobi's unit. He found the absolute electromotive force of a Daniell's element, in the mean out of several measurements,

$$
=10 \cdot 258 \times 10^{10}\left(\frac{\text { millim. } \cdot^{\frac{3}{2}} \text { milligr. } .^{\frac{1}{2}}}{\text { sec. }^{2}}\right) .
$$

This result is proportional to the resistance taken as the basis of the measurement; the error committed in measuring this resistance enters into the derived value of the electromotive force.

From a long series of absolute measurements of the electromotive forces of the Daniell element, the details of which shall be related in another place, I have found that the lowest value of the electromotive force of the Daniell element in absolute electromagnetic measure is

$$
10 \cdot 96 \times 10^{10}\left(\frac{\text { millim. } .^{\frac{3}{2}} \text { milligr. } .^{\frac{1}{2}}}{\text { sec. }^{2}}\right),
$$

that the absolute value of the electromotive force of a Daniell's element of the form usually employed is

$$
11 \cdot 30 \times 10^{10}\left(\frac{\text { millim. }^{\frac{3}{2}} \text { milligr. } .^{\frac{1}{2}}}{\text { sec. }^{2}}\right),
$$

and that the highest value of the electromotive force of a Daniell's element amounts to

$$
11.54 \times 10^{10}\left(\frac{\text { millim } .^{\frac{3}{2}} \text { milligr. } .^{\frac{1}{2}}}{\text { sec. }^{2}}\right)
$$

* Pogg. Ann. vol. ci. p. 517. 
Which form of Daniell's element Bosscha used, unfortunately he does not state; but we may assume as extremely probable that he made use of the form ordinarily employed, to which, according to my measurements, belongs the absolute electromotive force

$$
11 \cdot 30 \times 10^{10}\left(\frac{\text { millim } \cdot^{\frac{3}{2}} \text { milligr. } .^{\frac{1}{2}}}{\text { sec. }^{2}}\right)
$$

This value is greater, in the ratio of $1 \cdot 1016$ to $1 \cdot 0000$, than that deduced by Bosscha. Now, supposing that Bosscha has carried out his measurements free from error (a supposition which of course cannot be rigorously correct), then the absolute value of the resistance taken by him as the basis of his measurements, $i$. $e$. the absolute value found by W. Weber for Jacobi's unit, would be $10 \cdot 16$ per cent. too little.

This calculation of the error is based on two somewhat uncertain assumptions, briefly indicated above. On this account it is a great advantage that an error in W. Weber's determination of the absolute resistance of Jacobi's unit, in the same direction and of the same order of magnitude, can be deduced in quite another way. According to $W$. Siemens the ratio of Jacobi's resistance-unit to Siemens's is $=0 \cdot 6618$. From our numerous and multifariously varied measurements the absolute value of the Siemens unit is $0.9550 \times 10^{10}\left(\frac{\text { millim. }}{\text { sec. }}\right)$. Accordingly the absolute value of Jacobi's resistance-unit would be, from our measurements, $0.6320 \times 10^{10}\left(\frac{\text { millim. }}{\text { sec. }}\right)$; while M. Wilh. Weber found only $0.598 \times 10^{10}\left(\frac{\text { millim. }}{\text { sec. }}\right)$-that is, a value about 6 per cent. less than that found by us.

Hence the absolute measurement by M. W. Weber of Jacobi's resistance-unit has turned out certainly from 6 to 10 per cent. too little.

[To be continued.]

XIX. Crystallographic Notes.

By W. J. Lxwis, M.A., Fellow of Oriel College, Oxford*.

$D^{\mathrm{R} . \text { HUGO MÜLLER had the goodness, some time ago, }}$ Quercite and Inosite, which he had obtained from new sourcesthe former from the leaves of the dwarf-palm (Chamcerops humilis), and the latter from cochineal.

* Communicated by the Crystallological Society, having been read October 26, 1877. 